\title{
Frecuencia de prescripciones de medicaciones sintomáticas y antibióticos en menores de 5 años, en dos centros de atención pública y privada
}

\author{
Symptomatic medication and antibiotic prescription \\ frequency in children under 5 years of age, in two public \\ and private care centers
}

\author{
Delia Melissa Mancuello', Laura Godoy Sánchez²
}

\section{RESUMEN}

Introducción: existen factores que conducen a los pediatras a prescribir medicaciones "sintomáticas" y antibióticos, orientados a reducir síntomas y la ansiedad de los padres, práctica que no es inocua, porque algunas pueden producir daño. Objetivo: Determinar frecuencia de prescripciones de medicaciones sintomáticas y antibióticos, en consultas pediátricas de menores de 5 años de dos centros de atención, público y privado. Materiales y Métodos: Estudio observacional, descriptivo con componente analítico, prospectivo, transversal. Se incluyeron menores de 5 años que acudieron a consultas del Hospital Regional de Pedro Juan Caballero y Clínica Privada del Niño de la misma ciudad, en septiembreoctubre de 2017. Los datos fueron analizados en SPSSv21. Variables principales: Edad, sexo, procedencia, centro de consulta motivo de consulta, medicamentos prescriptos. Resultados: Fueron incluidos 200 pacientes, 51.5\% (103) fueron del centro privado y $48.5 \%$ (97) del público. Mediana de edad: 16 meses, no hubo diferencias en relación al sexo. Motivos de consultas más frecuentes: Infección de vías aéreas superiores en privado (32\%) y público (26\%). El uso de Medicaciones sintomáticas fue: privado $32,4 \%(53 / 179)$, público $29,6 \%(53 / 179)$, recibió una sola medicación sintomática el 29,7\%(33/111) y $27,9 \%(31 / 111)$ respectivamente. La frecuencia de prescripción de antibióticos en el privado fue 2,5\%(5/179) y público 1,7\% (3/179). El 89.5\%(179/200) recibió algún tipo de medicación. Conclusión: Poco menos de la mitad utilizaron medicación sintomática y una tercera parte

\begin{abstract}
Introduction: there are factors that lead pediatricians to prescribe "symptomatic" medications and antibiotics, aimed at reducing symptoms and parental anxiety, a practice that is not harmless, because some can cause harm. Objective: To determine the frequency of prescriptions of symptomatic medications and antibiotics in pediatric consultations for children under 5 years of age in two care centers, public and private. Materials and methods: An observational, descriptive study with an analytical, prospective, cross-sectional component. Children under 5 years of age who attended consultations at the Pedro Juan Caballero Regional Hospital and the Private Children's Clinic of the same city, in SeptemberOctober 2017, were included. The data were analyzed in SPSSv21. Main variables: Age, sex, origin, consultation center, reason for consultation, prescribed medications. Results: 200 patients were included, 51.5\% (103) were from the private center and $48.5 \%$ (97) from the public. Median age: 16 months, there were no differences in relation to sex. Reasons for the most frequent visits: Upper airway infection in private (32\%) and public (26\%). The use of symptomatic medications was: private $32.4 \%$ (53/179), public $29.6 \%$ (53/179), received a single symptomatic medication $29.7 \%(33 / 111)$ and $27.9 \%(31 / 111)$ respectively. The frequency of prescribing antibiotics in the private sector was $2.5 \%(5 / 179)$ and the public $1.7 \%$ (3/179). 89.5\% (179/200) received some type of medication. Conclusion: Just under half used symptomatic medication and one third used antibiotics, with no
\end{abstract}

\footnotetext{
${ }^{1}$ Hospital Regional de Pedro Juan Caballero. Pedro Juan Caballero, Paraguay.

${ }^{2}$ Universidad Católica "Nuestra Señora de la Asunción”. Asunción, Paraguay.

Correspondencia: Laura Godoy Correo:legodoys@gmail.com

Conflicto de interés: Los autores declaran no poseer conflicto de interés.

Recibido: 02/09/2020 Aceptado:29/09/2020

Doi: https://doi.org/10.31698/ped.47032020008
}

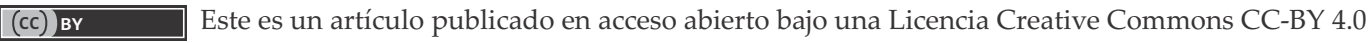


Se definió consulta privada como un servicio ambulatorio para pacientes con un médico pediatra de elección para acceder a atenciones médicas para diferentes tipos de diagnósticos pagando un costo determinado por la misma.

Medicación sintomática: es cualquier terapia médica de una enfermedad que solo afecta sus síntomas, no la causa subyacente por ejemplo: antipiréticos, descongestivos, analgésicos.

Cálculo del tamaño de la muestra: para conseguir una precisión del 3\% en la estimación de una proporción mediante un intervalo de confianza asintótico normal con corrección para poblaciones finitas al 95\% bilateral, asumiendo que la proporción esperada es de $90 \%$ y que el tamaño total de la población es de 240, fue necesario incluir 148 participantes en el estudio.

Procesamiento de los datos y plan de análisis: los datos fueron analizados en el sistema SPSSv21 utilizando estadística descriptiva e inferencial. Las variables cualitativas se expresaron en porcentajes y las cuantitativas en medias con desviación estándar o medianas de acuerdo a su distribución. Se consideró un error alfa menor $5 \%$. El protocolo fue aprobado por el comité de investigación y ética institucional con consentimiento informado verbal de los padres.

\section{RESULTADOS}

Durante el periodo de la investigación cumplieron los criterios de inclusión 200 pacientes, 103 de la consulta privada y 97 en la consulta pública los datos demográficos se resumen en la tabla 1.

Los motivos de consulta más frecuentes fueron en el sector privado y público respectivamente: Infección de Vías Aéreas Superiores 64/103 (62,1\%) vs 52/97 $(53,6 \%) \mathrm{p}=0,222$, Gastrointestinal 25/103(42,5\%) vs $30 / 97(30,9 \%) \mathrm{p}=0,312$ fiebre $13 / 103(12,6 \%)$ vs $15 / 97$ $(15,5 \%), p=0,563$.

Se determinó frecuencia de utilización de medicaciones y se determinaron las medicaciones más prescriptas. Las medicaciones fueron clasificadas en medicaciones sintomáticas, antibióticos y la combinación de ambos. Tabla 2.

Tabla 1. Datos demográficos de los pacientes que consultaron en la Clínica privada del niño y el Hospital Regional de Pedro Juan Caballero. N=200.

\begin{tabular}{|c|c|c|c|c|c|}
\hline \multirow[t]{2}{*}{ Edad (Meses) } & \multicolumn{2}{|c|}{$\begin{array}{c}\text { Mediana } \\
16 \\
\text { (P25:8 P75:36) }\end{array}$} & & & \multirow{4}{*}{$p$} \\
\hline & & rado & & lico & \\
\hline \multirow[t]{2}{*}{ Consultas } & $N$ & $\%$ & $\mathbf{N}$ & $\%$ & \\
\hline & 103 & 51.5 & 97 & 48.5 & \\
\hline Sexo & $N$ & $\%$ & $\mathrm{~N}$ & $\%$ & \\
\hline Masculino & 57 & 28.5 & 40 & 20 & \\
\hline Femenino & 46 & 23 & 57 & 28.5 & $0.064 *$ \\
\hline \multicolumn{6}{|l|}{ Procedencia } \\
\hline Urbana & 87 & 43.5 & 70 & 35 & \\
\hline Rural & 16 & 8 & 27 & 13.5 & $0.052 *$ \\
\hline
\end{tabular}

*Chi cuadrado de Pearson

Tabla 2. Uso de Medicaciones en pacientes que consultaron en la Clínica Privada del Niño y el Hospital Regional de Pedro Juan Caballero. $n=179$

\begin{tabular}{|c|c|c|c|c|c|}
\hline \multirow[t]{2}{*}{ Uso de Medicación } & \multicolumn{2}{|c|}{ Privado } & \multicolumn{2}{|c|}{ Público } & \multirow[t]{2}{*}{$\mathbf{p}$} \\
\hline & $N$ & $\%$ & $N$ & $\%$ & \\
\hline $\mathrm{Si} *$ & 95 & 47.5 & 84 & 42 & $0.194 * *$ \\
\hline Antibióticos & 5 & 2.8 & 3 & 1.7 & $0.585^{* *}$ \\
\hline $\begin{array}{l}\text { Antibióticos + } \\
\text { Medicación } \\
\text { sintomática }\end{array}$ & 32 & 17.8 & 28 & 15.6 & $0.960 * *$ \\
\hline $\begin{array}{l}\text { Medicaciones } \\
\text { Sintomáticas }\end{array}$ & 58 & 32.4 & 53 & 29.6 & $0.779 * *$ \\
\hline
\end{tabular}

Analizando el número de medicaciones sintomáticas indicadas, recibieron una sola medicación sintomática en el centro privado 33/95 (34,7\%) y en el público $31 / 84(36,9 \%)$, en tanto que más de una medicación sintomática 25/95 (26,3\%) y 22/84 (26,1\%) respectivamente.

\section{DISCUSIÓN}

En el presente estudio la frecuencia de uso de medicaciones tanto sintomáticas como antibióticos fue alta y en la misma proporción en los centros público y privado. En ambos sectores la mayoría procedía de zonas urbanas. Fue elevada la prescripción conjunta de antibióticos y medicaciones sintomáticas. 
antibióticos, sin diferencias entre el centro público y privado. Menos de un cuarto de los pacientes de ambos sectores utilizaron más de una medicación sintomática.

Palabras claves: Terapéutica, antibacteriano, pediatría, consulta pública.

\section{INTRODUCCIÓN}

En una consulta pediátrica, tanto pública como privada, ante un niño afecto de un proceso agudo, el principal objetivo es llegar en lo posible a un diagnóstico etiológico y establecer un tratamiento específico. En muchas ocasiones, el entorno familiar no tiene el mismo objetivo, sino más bien, demandan soluciones para los síntomas derivados de procesos agudos no graves o afecciones propias de la infancia; el hecho de asistir a la consulta, en muchos casos, provocan trastornos laborales a los padres, actitudes vinculadas a factores de la sociedad actual ${ }^{(1)}$.

El pediatra no debería indicar medicación alguna para calmar la ansiedad de los padres que acuden con su niño enfermo sino porque realmente lo necesite. Muchos factores conducen a algunos pediatras a la prescripción de las medicaciones llamadas "sintomáticas," así como de antibióticos orientados a la reducción de los síntomas del niño y la ansiedad de los padres, con lo cual se podría lograr la satisfacción de los mismos. Sin embargo esta práctica no es inocua, porque muchas de estas medicaciones son capaces de producir daño al paciente, pudiendo en el caso de los antimicrobianos, convertirse en portadores de bacterias resistentes $^{(2)}$. Aunque la mayoría de las medicaciones sintomáticas son relativamente seguras, el uso indebido puede llevar a la ingesta de cantidades excesivas e incluso a situaciones sobredosis con las consecuencias que esto conlleva, teniendo en cuenta lo mencionado anteriormente, algunos países ya han restringido el acceso a varios medicamentos de venta libre al introducir una categoría intermedia de medicamentos de farmacia, es decir sólo pueden ser comprados en farmacias o con receta ${ }^{(3)}$. Al respecto en el Paraguay ya se ha tomado esta medida en relación con los antibióticos. differences between the public and private centers. Less than a quarter of the patients in both sectors used more than one symptomatic medication.

Keywords: Therapeutic, antibacterial, pediatrics, public consultation.

El objetivo del presente trabajo fue determinar la frecuencia de las prescripciones de medicaciones sintomáticas y antibióticos en las consultas pediátricas de menores de 5 años en dos centros de atención, pública y privada de la cuidad de Pedro Juan Caballero capital del Departamento de Amambay.

\section{MATERIALES Y MÉTODOS}

Diseño y lugar del estudio: Estudio observacional, descriptivo, prospectivo de corte transversal con componente analítico, llevado a cabo en el Hospital Regional de la ciudad de Pedro Juan Caballero y en la Clínica Privada del Niño de la ciudad de Pedro Juan Caballero, Departamento de Amambay, Paraguay en los meses de septiembre y octubre del 2017.

Población y reclutamiento: por medio de un muestreo no probabilístico de casos consecutivos, fueron incluidos en el estudio niños menores de 5 años de edad que acudieron al consultorio del Hospital Regional y de la Clínica privada del Niño de la ciudad de Pedro Juan Caballero. Fueron excluidos los pacientes que requirieron internación, con comorbilidades y que hayan tenido dificultades en la comunicación. Se confeccionó un cuestionario con preguntas abiertas y cerradas, que los padres o encargados de los pacientes debían llenar previo consentimiento informado verbal al salir de la consulta. Se observaron también las recetas para la recolección de las medicaciones indicadas.

Variables estudiadas: Edad, Sexo, Procedencia, Centro de consulta, Motivo de Consulta, Medicamentos prescriptos. 
El uso de medicaciones sintomáticas fue elevado en ambos centros sin diferencias significativas, sin embargo es ligeramente menor en el Hospital Regional a la reportada por un estudio hecho en el Hospital General Pediátrico Niños de Acosta Ñu por Godoy y colaboradores en el año 2009 que incluyó 202 pacientes con el objetivo de describir el uso de prescripciones sintomáticas que fue del $91 \%{ }^{(4)}$ En otro estudio realizado en España por Blanco G, en el año 2013 se encontró que el 67,5\% de los pediatras prescriben antitusígenos ${ }^{(5)}$. Teniendo en cuenta los estudios mencionados podemos notar que las medicaciones sintomáticas son muy utilizadas en las consultas pediátricas, lo que nos lleva a reflexionar sobre el uso racional de estos medicamentos por los efectos colaterales que podrían causar, algunas de ellas pueden ser dermatológicas como las descritas por Gorrín y col en un estudio retrospectivo del Hospital Pediátrico de San Miguel del Padrón, Cuba de octubre de 2014 a 2016 que incluyó 106 pacientes, donde las reacciones exantemáticas fueron las más frecuentes en $64,2 \%$ siendo el 'fármaco más común las penicilinas en $46,3 \%{ }^{(6)}$. Poco menos de un tercio de los niños, tanto del sector público como privado, recibieron una sola medicación sintomática y un cuarto de ellos, más de una medicación en comparación al estudio realizado del Hospital General Pediátrico Niños de Acosta Nu mencionado previamente, donde el $52 \%$ recibió solo una medicación sintomática y el $48 \%$ más de una medicación ${ }^{(4)}$.

La Infección de las vías aéreas superiores seguidas de enfermedades gastrointestinales y fiebre fueron los motivos de consulta más frecuentes en ambos centros, lo que difiere de un estudio realizado en Hospital Infantil Roberto del Rio en 2007 cuyo principal motivo fue síndrome febril $(45,4 \%)$, respiratorio $(18,4 \%)$, digestivo $(16,2 \%)$ y los medicamentos sintomáticos se prescribieron en un alto porcentaje, revelando esto, que se trató más la sintomatología que la causa de los motivos de consulta $^{(7)}$.

En ocasiones los padres asisten a la consulta con la intención de que el médico le prescriba antibióticos, en un estudio realizado por Zeru y col en el año 2018 en el que evaluaron el conocimiento y la práctica de los padres sobre el uso de antibióticos para las infecciones del tracto respiratorio superior en niños, en las instituciones de salud de la ciudad de Aksum, en el norte de Etiopía, encontraron que 59,1\% pidió a los médicos que prescribieran antibióticos y 12,8\% de los padres no siempre siguió los consejos de los médicos sobre el uso de antibióticos ${ }^{(8)}$. En cuanto al uso de los mismos, en el presente trabajo, se encontró prácticamente la misma proporción en ambos sectores y con mayor frecuencia asociados a medicación sintomática. Menos de la mitad de los pacientes recibieron tratamiento antibiótico, podría decirse que 4 de cada 10 lo utilizaron en ambos centros, datos similares a un estudio multicéntrico realizado en España Castilla y León por Vázquez y colaboradores en el año 2010, que demostró un descenso en los últimos 3 años demostrando su uso total 20,7 dosis diarias, definidas por 1000 habitantes/día ${ }^{(9)}$. En otro estudio realizado por Di Martino y col en el año 2015 en la región de Lazio, Italia, la prevalencia fue mayor en el grupo etario de cero a cinco años con $58 \%$, si bien esa población fue también mucho mayor (271,780 niños), también se encontró que la prevalencia de prescripción era del $40 \%$ para los médicos generales y del $35 \%$ para los pediatras, lo que no se discriminó en este trabajo ${ }^{(10)}$. En tanto que en Ciudad del Este, Paraguay, en el año 2014, Yegros y colaboradores encontraron uso inapropiado de antibióticos en $30 \%{ }^{(11)}$. En el presente estudio no se buscó la adecuación o no de la prescripción de los antibióticos en particular.

En relación a los centros públicos y privados, no se encontraron diferencias en la frecuencia de uso de medicaciones sintomáticas o de los antibióticos al igual que un trabajo realizado por Uda y colaboradores que analizaron la prescripción de antibióticos en 3 áreas de Japón desde 2013 a 2016 y encontraron que el número total de recetas de antimicrobianos por habitante pediátrico fue más bajo en el área $\mathrm{A}$ y más alto en el área $\mathrm{C}$ y que el número de recetas de clínicas privadas de pediatría y otorrinolaringología fue casi el mismo en cada una delas 3 áreas ${ }^{(12)}$.

Las medicaciones sintomáticas y antibióticas muchas veces pueden verse afectadas por las sugerencias de los padres, a veces de manera sustancial como se puede observar en un trabajo realizado por Zanasi y col que realizó una encuesta a 300 pediatras (todos 
miembros del Observatorio Nacional Italiano sobre Salud de la Infancia y la Adolescencia) cuyos hallazgos muestran que la mayoría de los pediatras (92\%) prescriben terapia en la tos aguda, además encontraron abuso de la terapia con aerosoles $(26 \%$ en tos aguda y $38 \%$ en tos crónica) y de prescripción de antibióticos (22\% en tos aguda y $42 \%$ en tos crónica $)^{(13)}$. Por la presión de los padres, podría haber diferencias sobre todo en los centros privados, lo que no se constató en esta población de estudio.

\section{REFERENCIAS}

1. García-Mozo R, Alonso-Álvarez A. Tratamiento sintomático de síntomas frecuentes en pediatría. Bol Pediatr. 2006;46:321-26.

2. Peñaranda Pérez I. Uso racional de antibióticos. Rev. bol. ped. [Internet]. 2011[citado 26 Ago 2020]; 50(3):150-151. Disponible en: http://www.scielo.org.bo/scielo.php?scri pt=sci_arttext\&pid=S1024-06752011000300001\&lng=es

3. Sobczak Ł, Goryński K. Pharmacological Aspects of Over-the-Counter Opioid Drugs Misuse. Molecules. 2020;25(17):3905. doi: http://dx.doi.org/10.3390/molecules 25173905

4. Godoy Sánchez LE, Mesquita M, Domínguez S. Uso de la Medicación Sintomática en la consulta pediátrica. Pediatr (Asunción). 2009;36(3):195-201.

5. Blanco Guzmán E, Ruano López A, Ugarte Libano R. Prescripción de antitusígenos por pediatras de Atención Primaria del País Vasco. Rev Pediatr Aten Primaria [Internet]. 2013 [citado 26 Ago. 2020]; 15(59):e85-e88. Disponible en: http://scielo.isciii.es/scielo.php?script=sci_ arttext\&pid=S1139-76322013000400006\&lng=es.http://dx. doi.org/10.4321/S113976322013000400006

6. Gorrín M, Rodríguez R, Villalobos S, González C. Comportamiento de las reacciones adversas a medicamentos en el Hospital Pediátrico San Miguel del Padrón. Folia Dermatológica Cubana [Internet]. 2017 [citado 30 Ago. 2020];11(2):e56.

7. Méndez EB, Herrera LP, Guerra MH, Dattas L, Muñoz $\mathrm{HB}$, Velasco BJ. Estructura de la consulta pediátrica en el Servicio de Urgencia: Hospital Infantil Roberto del Río. Rev. chil. pediatr. 2005; 76(3):259-265. doi: http://dx.doi.or $\mathrm{g} / 10.4067 / \mathrm{S} 0370-41062005000300004$

\section{CONCLUSIONES}

Poco menos de la mitad utilizaron medicación sintomática y una tercera parte antibióticos, sin diferencias entre el centro público y privado.

Menos de un cuarto de los pacientes de ambos sectores utilizaron más de una medicación sintomática.

8. Zeru T, Berihu H, Buruh G, Gebrehiwot H, Zeru M. Parental knowledge and practice on antibiotic use for upper respiratory tract infections in children, in Aksum town health institutions, Northern Ethiopia: a crosssectional study. Pan Afr Med J. 2020;35:142. doi: http://dx.doi.org/10.11604/pamj.2020.35.142.17848

9. Vázquez ME, Eiros J, Martín F, García S, Bachiller RM, Vázquez MJ. Prescripción de antibióticos a la población pediátrica de Castilla y León en la última década: tendencias, fluctuaciones estacionales y diferencias geográficas. Rev Esp Quimioter. 2012; 25(2):139-146.

10. Di Martino M, Lallo A, Kirchmayer U, Davoli M, Fusco D. Prevalence of antibiotic prescription in pediatric outpatients in Italy: the role of local health districts and primary care physicians in determining variation. A multilevel design for healthcare decision support. BMC Public Health. 2017;17(1):886. doi: http://dx.doi.org/10.118 6/s12889-017-4905-4

11. Yegros Martínez PE, Samudio M. Uso inapropiado de antibióticos en pobladores Ciudad del Este, Paraguay. An. Fac. Cienc. Méd. (Asunción) [Internet]. 2014 [citado 30 Nov. 2019]; 47(1):61-72. Disponible en: http://scielo.iics. una.py/scielo.php?script=sci_arttext\&pid=S181689492014000100005\&lng=en

12. Uda K, Kinoshita N, Morisaki N, Kasai M, Horikoshi Y, Miyairi I. Targets for Optimizing Oral Antibiotic Prescriptions for Pediatric Outpatients in Japan. Jpn J Infect Dis. 2019;72(3):149-159. doi: http://dx.doi.org/10.78 83/yoken.JJID.2018.374

13. Zanasi A, Morcaldi L, Cazzato S, Mazzolini M, Lecchi, M, Morselli-Labate A. et al. Survey on attitudes of Italian pediatricians toward cough. Clinicoecon Outcomes Res. 2017;9:189-199. doi: http://dx.doi.org/10.2147/CEOR.S129696 